\title{
Applying of Fuzzy Logic Interface in Nylon Fiber Production
}

\author{
Kunal Singha $^{1, *}$, Subhankar Maity ${ }^{1}$, Mrinal Singha ${ }^{2}$ \\ ${ }^{1}$ Department of Textile Technology, Indian Institute of Technology, Delhi, India \\ ${ }^{2}$ Department of Pharmaceutical Chemistry, CU Shah College of Pharmacy \& Research, Gujarat, India
}

\begin{abstract}
The nylon fiber by its high strength and modulus is becomes a potential option for many applications like general technical textile, high performance uses, nanotechnology material as a reinforced composite formulation and nanocrisper. The production of nylon fiber can be easily predicted and perform at an accurate level of energy saving with the help of using fuzzy logic interface. The circulation of hot/cold oil flow which has normally used as the fuel to increase the temperature of the nylon fiber reactor can be precisely checked by fuzzy logic data. The pressure and temperature of the reactor can be also controlled by this process of adding fuzzy intelligence or simulation in the chamber of the nylon fiber production reactor.
\end{abstract}

Keywords Nylon Fiber, Fuzzy Logic Interface, Hot/Cold Oil Flow, Temperature, Pressure, Fuzzy Intelligence, Simulation

\section{Introduction}

Fuzzy logic is a form of multi-valued logic derived from fuzzy set theory to deal with reasoning that is approximate rather than precise. Several studies ${ }^{1,2,3,4}$ are conducted using a fuzzy approach in order to simulate, predict and evaluate textile structure properties. In fact, several advantages make fuzzy logic theory among the tools of forecast which are most used by researchers. Altinoz ${ }^{1}$ suggests that fuzzy logic is an enabling technology that can be used to capture expertise and compute using linguistic rules for supplier selection. Usually, the fuzzy logic method is based on four essential steps. First, fuzzification consists to convert the feature values of input and output parameters. Second, design of the fuzzy rules to implement the model for prediction. Third, the fuzzified values are then inferred to provide decisions by the inference engine with the support of the fuzzy rule base. Finally selection by defuzzification converts fuzzy sets into a crisp value ${ }^{3}$. There are five built-in methods supported: centroid (used on our study), bisector, medium-maximum (the average of the maximum value of the output set), high-maximum and low-maximum. In this paper we deals with how to maximise production of nylon fiber by using fuzzy logic technique at a optimum energy supply.

Batch and semi-batch polymerization are typical of some processes such as PVC (poly vinyl chloride) and nylon

* Corresponding author:

kunalsingha28@gmail.com (Kunal Singha)

Published online at http://journal.sapub.org/fs

Copyright (C) 2012 Scientific \& Academic Publishing. All Rights Reserved production. The difficulty of measuring controlled variables, the existence of interactions, dead time and constraints, added to the nonlinear and mu ltivariable nature of the system, pose challenging problems for the control of poly merization reactors for the batch and semi-batch reactors in particular, additional difficulties arise concerning process variables, such as reactor temperature and pressure, which have to follow set-point trajectories to assure the quality of the final product. In some cases, control is achieved using an open loop control approach. Mainly we use fuzzy logic application in semi -batch process in case of nylon 6 polymerization $^{5,6}$.

\section{Fuzzy Control in Nylon 6 Semi Batch}

The process comprises a semi-batch reactor (V-tube) for the production of nylon $6\left(2.7 \mathrm{~m}^{3} \text { volu me }\right)^{5}$, equipped with stirrer and jacket, where hot oil or cold oil flow (not simu ltaneously) promotes heating or cooling of the reaction mass. One complete batch takes about $6 \mathrm{hr}$ to complete ${ }^{6}$. At the first stage- caprolactam and water are feed into the reactor. The reaction is endothermic initially and the reaction mass has to be heated for $3 \mathrm{hr}$ from the initial temperature $\left(90^{\circ} \mathrm{C}\right)$ up to $260^{\circ} \mathrm{C}$. At this point, the - caprolactam rings open, resulting in amino-caproic acid formation. Over the following $3 \mathrm{hr}$, the reactor temperature is maintained at $260^{\circ} \mathrm{C}$ and the polyaddition reaction (exothermic reaction) of the aminocaproic acid takes place. As a result the reaction mass becomes overheated causing liquid desorption from the free surface and desorption through bubble formation resulting in water vaporization and also vaporization of a small amount of caprolactam. Subsequently the initial 
pressure of 2 at $\mathrm{m}$ is increased for $3 \mathrm{hr}$ to reach the required 5 atm pressure and this is maintained for a further hour. After this a pressure reduction procedure starts up to reach atmospheric pressure. A vacuum pump is then started and depressurization begins for $1 \mathrm{hr}$, reaching $420 \mathrm{mmHg}$. The polymer is immediately discharged from the reactor, solidified with cool water and set out for drying and palletizing processes ${ }^{7,8,10}$

The main problem is here is that to maitain a proper temperature $\left(260^{\circ} \mathrm{C}\right)$ and pressure $(2 \mathrm{~atm})$ becouse of the control problem is typically a set-point tracking or servo problem and the reaching of these specified requirements is of great importance to assure the desired quality of the final product. we can solve this problem by using a flow of hot /cold oil through around the VK-tube used with the help of

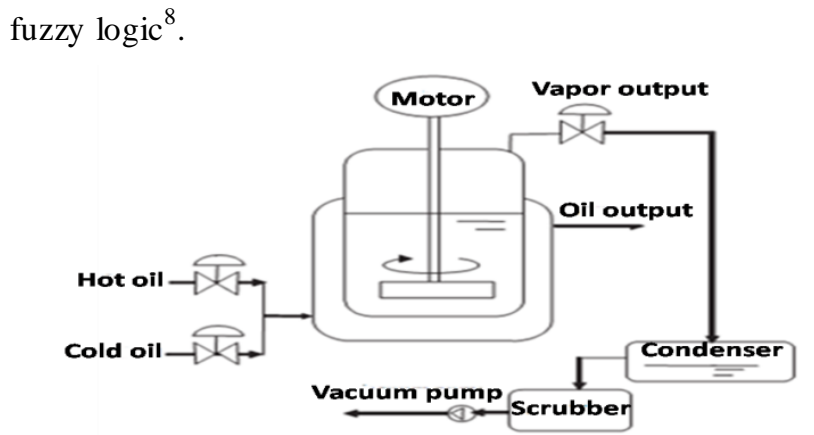

Figure 1. Semi-batch reactor for theproduction of nylon 6 (heating and cooling system $)^{8}$

\section{Temperature controller setpoint}

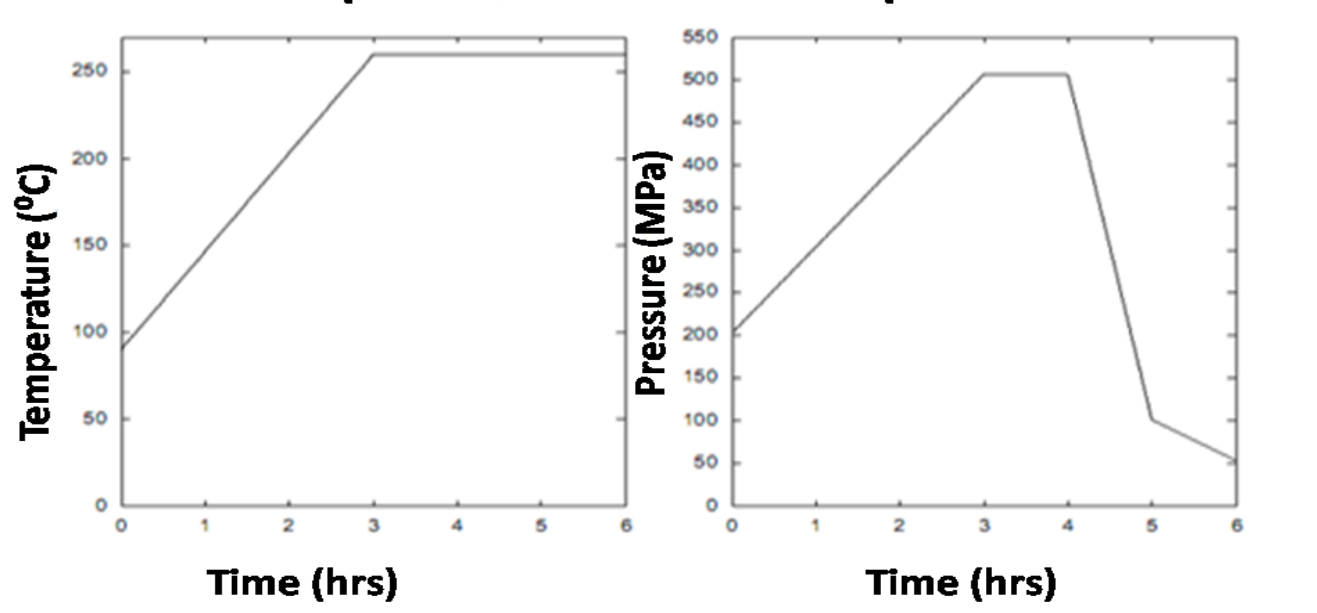

Figure 2. Set-point traject ories for temperat ure and pressure ${ }^{8}$

Generally we used three kind of fuzzy controllers as (a) Proportional fuzzy controller (b) PI fuzzy controller (c) PID (proportional,integral and derivartive) fuzzy controller and to apply the fuzzy logic we need the following assumption and notations firstly like;

\section{Kinetic and Mathematic Modeling}

The equations of the phenomenological model adopted in this work by uses the following assumptions ${ }^{9}$

- Heat transfer occurs from the hot oil inside the jacket to the reaction mass in the heating step and from the reaction mass to the cold oil in the cooling step.

- Water and - caprolactam vaporization from the reaction mass.

- Pressure build-up due to the heating process.

- Variable viscosity of the reaction medium during the batch and its effects on the mass and energy transfer coefficients.

- Mass and energy balances of the system. 
The following kinetic model was considered:

Ring opening reaction of the - caprolactam $\left(C_{1}\right)$ monomer, with the amino- caproic acid $\left(S_{l}\right)$ formation ( $W$ refers to water $)^{1,8}$

$$
\mathrm{C}_{1}+\mathrm{W} \leftrightarrow \mathrm{S}_{1}
$$

Polycondensation reaction $\left(S_{m}, S_{n}\right.$ and $S_{m+n}$ are polymer chains):

$$
\mathrm{S}_{\mathrm{m}}+\mathrm{S}_{\mathrm{n}} \leftrightarrow \mathrm{S}_{\mathrm{m}+\mathrm{n}}+\mathrm{W} \quad \mathrm{m}, \mathrm{n}=1,2,3, \ldots .
$$

Polyaddition reaction

$$
\mathrm{S}_{\mathrm{n}}+\mathrm{C}_{1} \leftrightarrow \mathrm{S}_{\mathrm{n}+1}
$$

Oligo mer ring opening reaction

$$
\mathrm{C}_{\mathrm{m}}+\mathrm{W} \leftrightarrow \mathrm{S}_{\mathrm{m}} \quad \mathrm{m}=2, \ldots . .5
$$

Oligo mer polyaddition

$$
\mathrm{S}_{\mathrm{n}}+\mathrm{C}_{\mathrm{m}} \leftrightarrow \mathrm{S}_{\mathrm{n}+\mathrm{m}}
$$

By using the above five equations we can finally derived a error determining equation as follows:

$$
\mathrm{C}(\mathrm{k})-\mathrm{C}(\mathrm{k}-1)=\mathrm{K}_{\mathrm{c}}[\mathrm{E}(\mathrm{k})-\mathrm{E}(\mathrm{k}-1)]+\left[\mathrm{T} . \mathrm{K}_{\mathrm{c}} \cdot \mathrm{E}(\mathrm{k})\right] / \tau_{1}
$$

The PI-fuzzy controller considers error $\left(E_{i}\right)$ and error variation $\Delta E_{i}$ as inputs and the output is the variation in control action $\Delta C_{i . .}$ This controller is directly as sociated with the conventional PI controller, where;
C ( i ) controller output signal

[ $\mathrm{C}_{\mathrm{i}}$ ] concentration of -caprolactam (1) and cyclic dimmer (2) in liquid phase (Mol/kg mixture)

W water concentration in liquid ( mol h-1)

S1 amino-caproic acid concentration in liquid ( $\mathrm{mol} / \mathrm{kg})$

$\zeta \quad$ dimensionless time const ant

So rewrittening the eqn. (6) we can get:

$$
\Delta \mathrm{C}(\mathrm{k})=\mathrm{K}_{\mathrm{c}} . \Delta \mathrm{E}(\mathrm{k})+\left[\mathrm{T} . \mathrm{K}_{\mathrm{c}} / \tau_{1}\right] \mathrm{E}_{\mathrm{k}}
$$

where; error $=\mathrm{E}_{\mathrm{i}}$, error deviation $=\Delta \mathrm{E}_{\mathrm{i}}$, and control action variation $=\Delta C_{i}$; but we should remember that this all entities are actually depends on some other process parameters which is very complex to understanding. So by controlling the generic subset rules in PID controller like $\zeta, \Delta \mathrm{E}(\mathrm{k})$ we can control $\Delta \mathrm{C}(\mathrm{k})$, hence we can gain the required temperature and pressure in the VK-tube reactor. The PID controller action diag ram has shown as per below ${ }^{1,11,12}$.

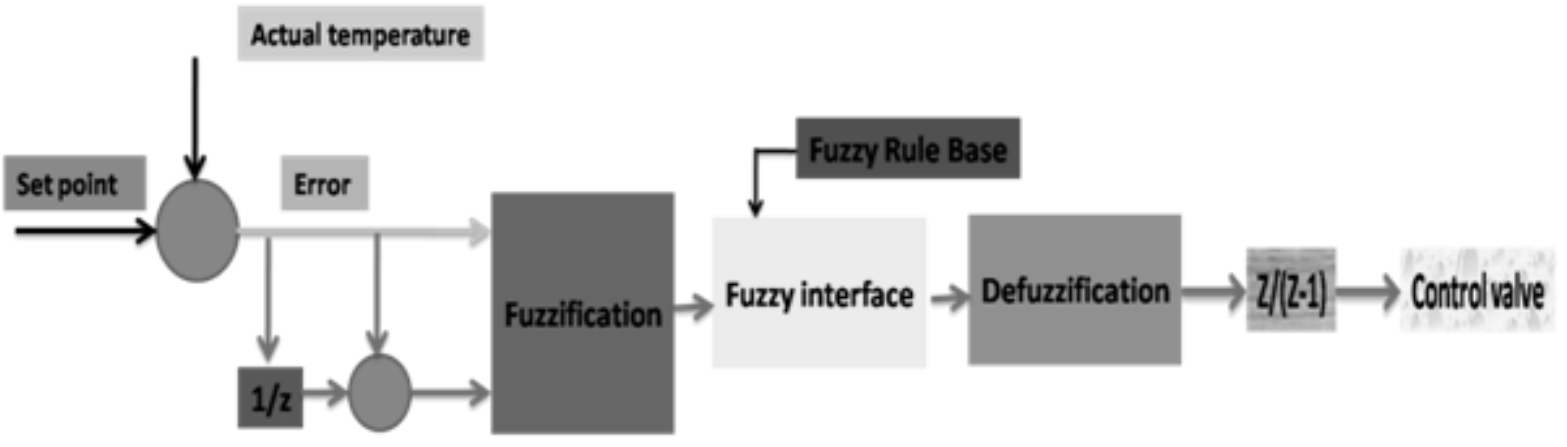

Figure 3. PI fuzzy controller diagram ${ }^{1}$

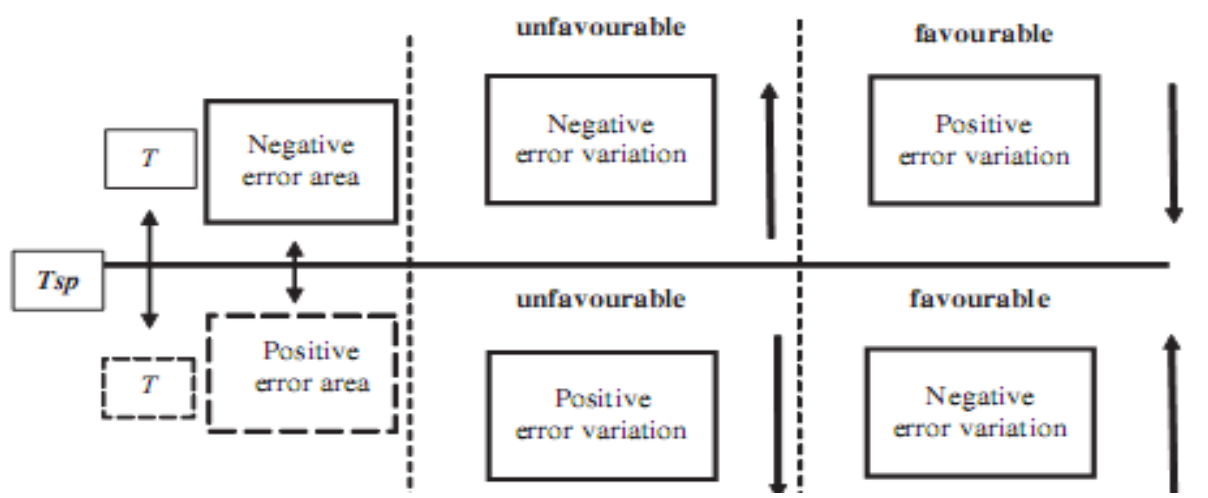

Figure 4. Configurations for the error and the error variation (temperat ure control) ${ }^{3}$

\section{Fuzzy Control Design}

The fuzzy inference system adopted in this work is based on the Mamdani model based on human analy zing power for a given situation how far is closed to the real phenomenan. In case of controlling the temperature and pressure in nylon 6 polymerization we can get the follo wing errors like: Zero error, Positive error, Negative error, Zero error variation, Positive error variation, Negative error variation, Great control action variation, Small control action variation, Zero control actio $n$ variation $^{13,14,17}$. 
Table 1. Rules for the fuzzy cont rol of temperat ure ${ }^{13}$

\begin{tabular}{ccccccc} 
Rule & Error & $\begin{array}{c}\text { Oil flow } \\
\text { change }\end{array}$ & Condition & $\begin{array}{c}\text { Error variation } \\
\Delta \mathbf{E}(\mathbf{k})\end{array}$ & Conse quent & Con trol action variation $\Delta \mathbf{C}$ \\
\hline 1 & Zero & Hot oil & and & Zero & Then & $\Delta c$ is zero \\
2 & Positive & Hot oil & And & Positive & Then & $\Delta c$ is large opening \\
3 & Positive & Hot oil & And & Negative & Then & $\Delta c$ is small opening \\
4 & Positive & Cold oil & And & Zero & Then & $\Delta c$ is small opening \\
5 & Negative & Cold oil & And & Positive & Then & $\Delta c$ is large opening \\
7 & Negative & Negative & Cold oil & and & Negative & Then
\end{tabular}

Table 2. Rules for the fuzzy control of pressure ${ }^{13}$

\begin{tabular}{|c|c|c|c|c|c|c|}
\hline Rule & Error $\mathbf{E}(\mathbf{k})$ & $\begin{array}{l}\text { Oil flow } \\
\text { change }\end{array}$ & Condition & $\begin{array}{c}\text { Error } \\
\text { variation } \\
\Delta \mathrm{E}(\mathbf{k}) \\
\end{array}$ & Consequent & $\begin{array}{c}\text { Control action variation } \\
\Delta \mathrm{C}\end{array}$ \\
\hline 1 & zero & & & & & $\Delta c$ is zero \\
\hline 2 & positive & Hot oil & and & Zero & Then & $\Delta c$ is large closing \\
\hline 3 & positive & Hot oil & And & Positive & Then & $\Delta c$ is large closing \\
\hline 4 & positive & Hot oil & And & Negative & Then & $\Delta c$ is small closing \\
\hline 5 & Negative & Cold oil & And & Zero & Then & $\Delta c$ is small opening \\
\hline 6 & Negative & Cold oil & And & Positive & Then & $\Delta c$ is large closing \\
\hline 7 & Negative & Cold oil & and & Negative & Then & $\Delta c$ is small opening \\
\hline
\end{tabular}

We can thus put some logistics gate ( 0 or 1 i.e. $0 / 1)$ to maintain our desired conditions of temperature and pressure.we can also show our results in a diagram as per below;
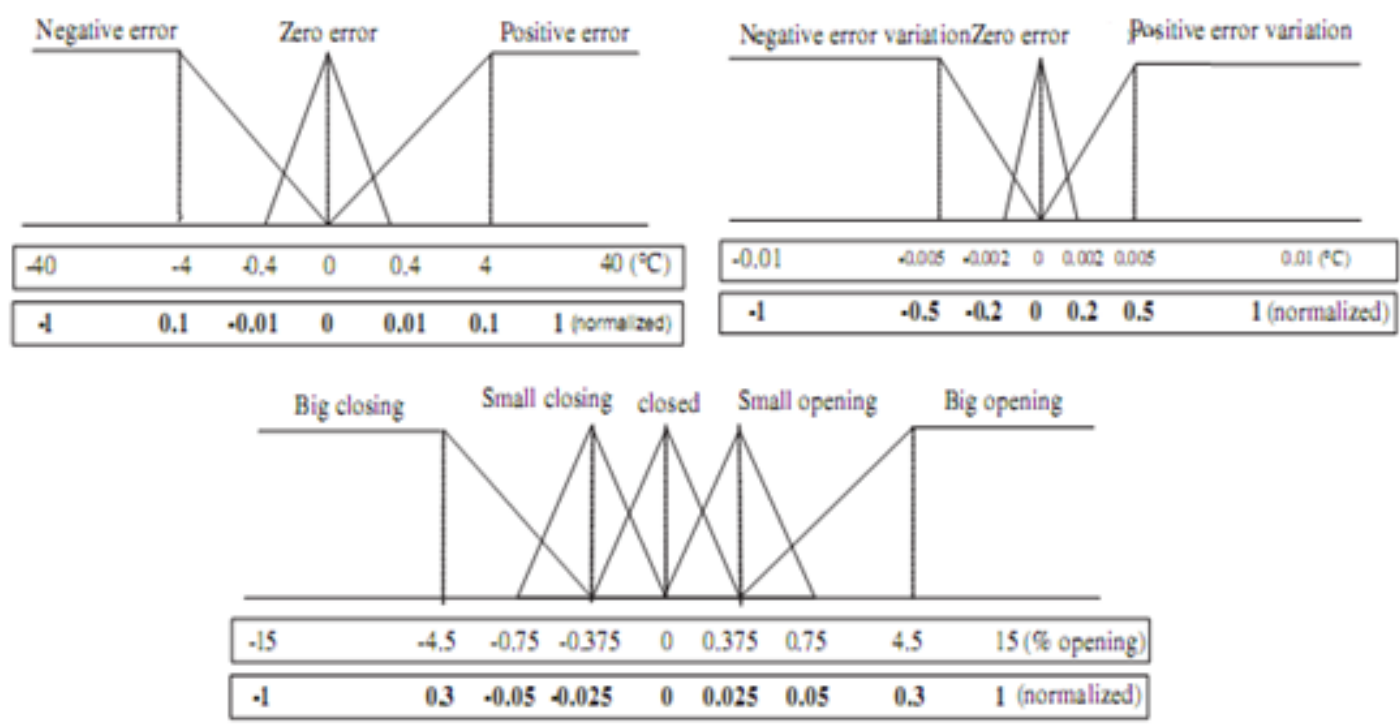

Figure 5. Membership functions of error, error variation and control action variation (temperat ure fuzzy control) ${ }^{15}$

And the required way for oil flowing technique is shown as below: 


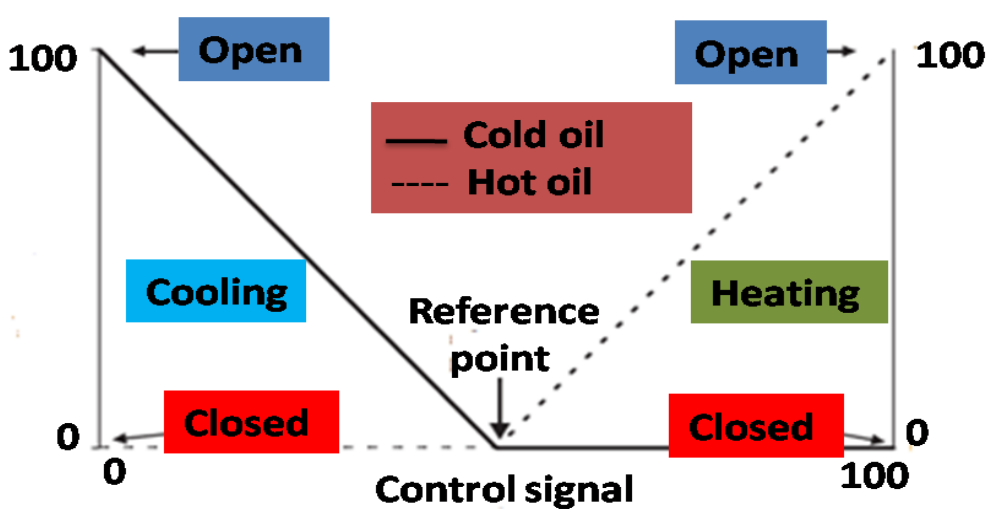

Figure 6. Split range configuration for oil flowing ${ }^{17}$

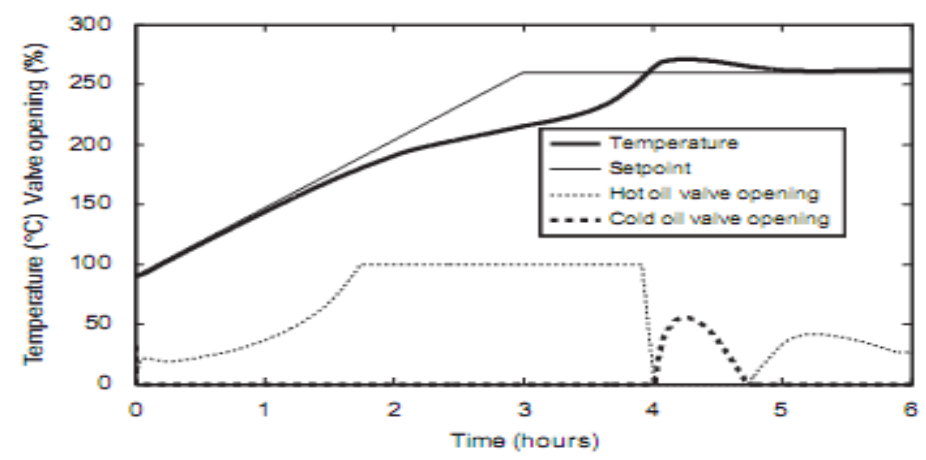

Figure 7. Temperature and manipulated variables (conventionalPID control) ${ }^{17}$

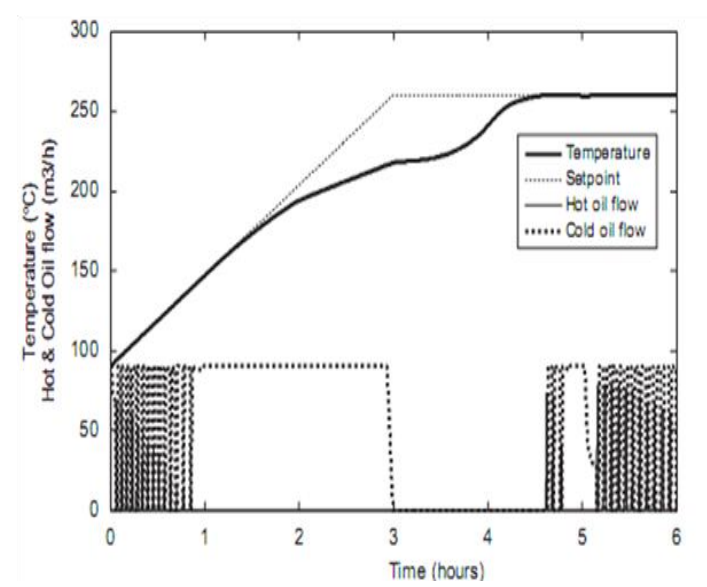

(a)

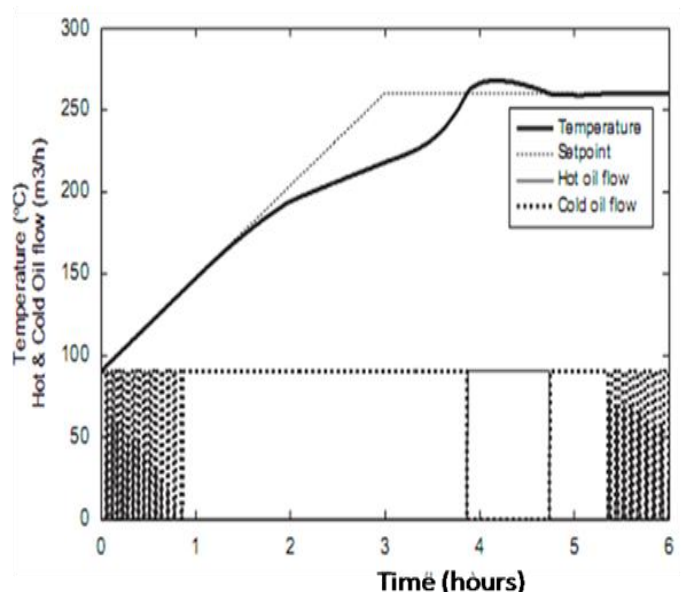

(b)

Figure 8. (a) Temperature and manipulated variables (PI-fuzzy control) (without anticipat ory action) and (b) Temperat ure and manipulated variable (PI-fuzzy control) (with anticipat ory action) ${ }^{16}$
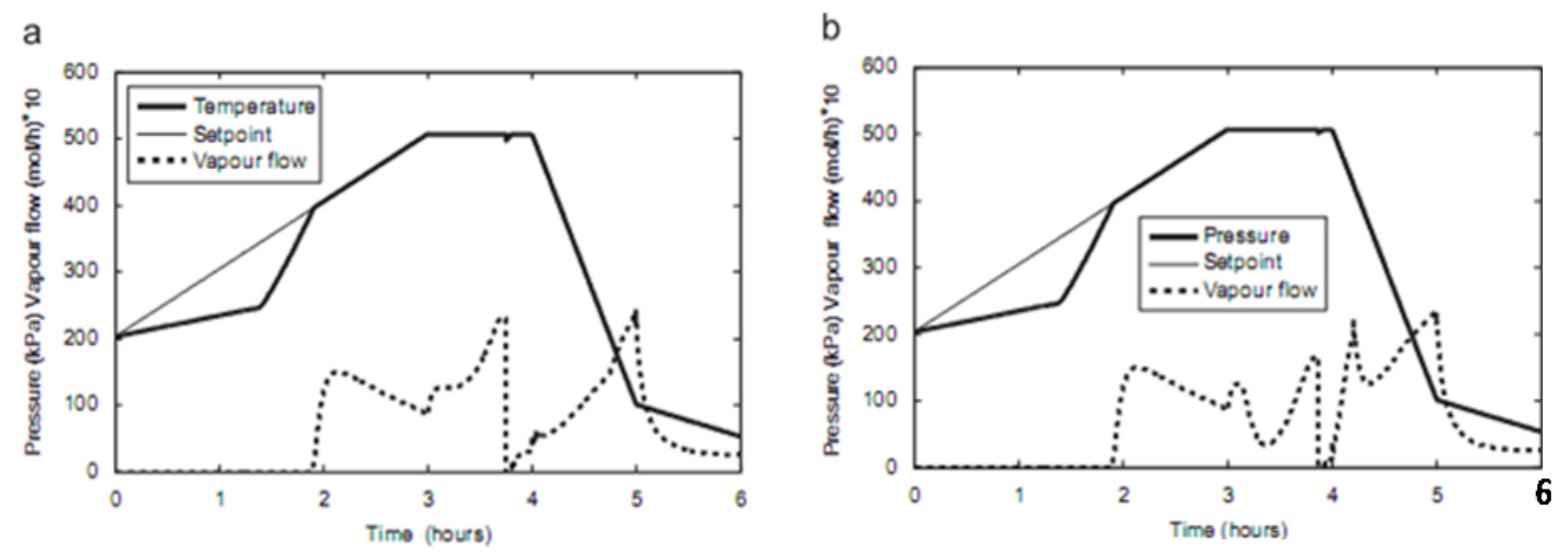

Figure 9. (a) Pressure and manipulated variable (PI-fuzzy control) (without anticipatory action) and (b) pressure and manipulated variable (PI-fuzzy control) (with anticipat ory action, Table 2$)^{18}$ 


\section{Concluding Remarks}

Nylon is an important fiber in today's world because of its highly rigid but suitable structure for most of the processing later. In this current review we have predicted the best energy saving model for nylon 6 polymerization spinning process by applying fuzzy modeling ${ }^{192021,22}$. Indeed, the results also show that the change of fuzzy membership function affects the prediction accuracy of the rate of polymerization. Compared to experimental results, it may be concluded that, overall, theoretical models using fuzzy technique are marked by relatively acceptable correlation coefficients especially using triangular membership function $^{23,24}$. Traditional methods are limited to evaluating bagging appearance by single parameters such as oil flow, rate of the reaction etc. which cannot represent the abundant information given by the appearance reactor system ${ }^{11,23}$. The error values between analytical and experimental results within and without fuzzy system show the effectiveness of the fuzzy forecast model ${ }^{25,26}$. Thanks to the fuzzy interface technique, we could make possible the forecast of the value of the nylon 6 reactor processing exactly during the manufacturing of the polymer.

\section{REFERENCES}

[1] Altinoz, C. \& Winchester, S. A Fuzzy Approch to Supplier Selection, J. Text. Inst. 92(2), 155- 167 (2001).

[2] Jaouachi, B., Mohamed B.H., Sahnoun M. \& Sakli F. Evaluation of wet pneumatically splice delastic denim yarns with fuzzy theory, J. Tex. Inst. 101(2), 111-119 (2010).

[3] Cox, E. Fuzzy logic for business and industry, M A: Charles River Media, 39-59 (1995).

[4] Hyung, T., Sung, H., Sook, R., Jae, Y. \& Seong, H. Detecting fabric defects with computer vision and fuzzy rule generation part II: defect identification by a fuzzy expert system. Textile Res. J. 71(7), 563-573 (2001).

[5] Altinten, A., Erdo gan, S., Hapo glu, H. \& Alpbaz M. Comput. Chem. Eng. 27, 1031-1040 (2003).

[6] Antunes, A.J.B., Pereira, J.A.F.R. \& Fileti, A.M.F. Comput. Chem. Eng. 30, 268-276 (2005).

[7] R.Babuska, R. \& Verbruggen, H.B. Control Eng. Practice 4, 1593-1606 (1996).

[8] Chen, B., Liu, X. \& S.Tong Solitons and Fractals 37, 894-901
(2008).

[9] Guerra, T.M., Kruszewski, A., Vermeiren, L. Tirmant, H., Fuzzy Sets and Systems 157, 1248-1259 (2006).

[10] Carothers, W.H.U.S. Patent 2, 130, 523 (1938).

[11] Gupta, A. Gupta, S.K. Gandhi, K.S., Mehta, M.H., Padh, M.R., Soni, A.V. \& Ankleswaria, B.V. Chem.Eng.Comm.113, 63-89 (1992).

[12] Gupta, S.K. \& Kumar, A. Plenum,New York (1987).

[13] Hanai, T. Ohki, T. Honda, H. \& Kobayashi, T. Comput. Chem. Eng. 27, 1011-1019 (2003).

[14] Jantzen, J. Techical Report no 98-H-871, Technical University of Denmark, Department of Automation (1998).

[15] Sala, A., Guerra, T.M. \& Babuska, R. Fuzzy Sets and Sy stems $156,432-444$ (2005).

[16] Haggen muellerbaa, R., Dub, F., Fischera, J.E. \& Winey a, K.I. Interfacial in situ polymerization of single wall carbon nanotube/nylon 6,6 nanocomposites Department of Materials Science and Engineering, University of Pennsylvania, Philadelphia, PA 19104-6272, USA, Department of Chemical and Biomolecular Engineering, University of Pennsylvania, Philadelphia, PA 19104-6393, USA.

[17] King, P.J. \& Mamdani, E.H. Automatica 13, 235-242 (1977).

[18] Chen, B. \& Liu, X. Fuzzy Sets and Systems 146, 349-374 (2004).

[19] Li, C., Kuang, K., Karthik, R. \& Rao, S.S. Mater.Design 17, 235-244 (1996).

[20] Tai, K. \& Tagawa, T. Indust. Eng. Chem. Prod. Res. Develop. 22, 192-206 (1983).

[21] Wakabayashi, C., Embiruçu, M., Fontez, C. \& Kalid. R. Fuzzy control of a nylon polymerization semi-batch reactor; Escola Politecnica, Universidade Federal da Bahia, Rua Aristides Novis, 2 Federaçao, CEP-40210-630 Salvador, BA, Brazil.

[22] Tanaka, K. \& Wang, H.O. Fuzzy Control Systems Design and Analy sis, Wiley, New York, 2001

[23] Wajge, R.M., Rao, S. \& Gupta, S.K. Polymer 35, 3722-3734 (1994).

[24] Yabuki, Y., Nagasawa, T. \& MacGregor, J.F. Comput. Chem. Eng. 26 (2002) 205-212.

[25] Yoney ama, J. Fuzzy Sets and Sy stems 158, 115-134 (2007).

[26] Yu, F.M., Chung, H.Y. \& Chen, S.Y. Fuzzy Sets and Sy stems 140, 359-374 (2003). 